\title{
Mercado de Trabalho Cearense: Desemprego e Desempregados. Quem São Eles?
}

\author{
Luís Abel da Silva Filho ${ }^{1}$ \\ Silvana Nunes de Queiroz ${ }^{2}$ \\ Maria do Livramento Miranda Clementino ${ }^{3}$
}

\begin{abstract}
Resumo: A discussão acerca do desemprego ganhou relativa dimensão, notadamente a partir dos anos de 1990, quando se registraram as maiores taxas de desemprego aberto já vistas no Brasil. Ao longo dos anos 2000, a ruptura à tendência dos anos pretéritos começou a dar os primeiros sinais a partir de 2004, com redução contínua do desemprego no país. Destarte, é pretensão deste artigo analisar o desemprego no Ceará e na Região Metropolitana de Fortaleza (RMF). Os dados são da Pesquisa Nacional por Amostra de Domicílios (PNAD), do Instituto Brasileiro de Geografia e Estatística (IBGE). O recorte temporal analisado são os anos de 2001-2008. Como metodologia, além de uma revisão de literatura acerca do desemprego, utilizou-se o método de decomposição do desemprego, que permite através de técnicas de mensuração observar a participação de cada grupo na composição do desemprego total de uma região. O método de composição do desemprego permitiu observar que, ao longo dos anos analisados, ocorreu redução no estoque força de trabalho masculina, no estoque total de desempregados do estado, em detrimento da elevação do estoque feminino. Por outro lado, dinâmica oposta foi observada na RMF. Além do mais, constatou-se elevação da força de trabalho jovem com idade entre 15 a 24 anos no estoque total de desempregados, bem como redução da participação dos menos escolarizados (o a 4 anos de estudo) no desemprego total, tanto no estado quanto na RMF. As evidências, portanto, permitem inferir que o mercado de trabalho cearense é seletista, o que torna determinados grupos mais vulneráveis, como é o caso de mulheres, não brancos, jovens e residentes, notadamente na RMF.
\end{abstract}

Palavras-chave: Mercado de trabalho; Desemprego; Ceará; RMF. JEL: J70.

1 Mestre em Economia pela Universidade Federal do Rio Grande do Norte - UFRN. Pesquisador do Observatório das Metrópoles, núcleo da UFRN. Professor do Departamento de Economia da Universidade Regional do Cariri - URCA. E-mail: abeleconomia@hotmail.com

2 Doutora em Demografia pela Universidade Estadual de Campinas - UNICAMP. Professora Assistente do Departamento de Economia da Universidade Regional do Cariri - URCA. E-mail: silvanaqueirozce@yahoo.com.br 


\title{
Cearense Job Market: Unemployment and Unemployed. Who Are They?
}

\begin{abstract}
: the discussion gained relative size of unemployment, especially from the 1990s, when it registered the highest unemployment rates ever seen in Brazil. Over the year 2000, breaking the trend of years gone by began showing the first sings from 2004, with continued reduction of unemployment in the country. Thus, claims if this article is to analyze unemployment Ceará and Fortaleza metropolitan Region (RMF). Data are from the National Household Sample Survey (PNAD), the Brazilian Institute of Geographic and Statistic (IBGE). The time frame is considered the years 2001-2008. The methodology and a literature review of unemployment, we used the method of the compositions of unemployment, which allows measurement techniques by observing the share of each group in the composition of total unemployment in a region. The method composition of unemployment has observed that over the years analyzed, there was a reduction in the male labor supply, the total stock of unemployment in the estate, to the detriment of the stock increase female. On the other hand the opposite dynamic was observed in the RMF. Moreover, there was increase young workforce aged 12 to 24 years in the total stock of unemployment as well as reducing the participation of educated (o to years of schooling) in total unemployment, both in estate in the RMF. The evidence, therefore, by inferred that the labor market is selective Ceará, which makes certain vulnerable groups such as women, nonwhites and younger residents, especially in the RMF.
\end{abstract}

Keywords: labor market; Unemployment; Ceará; RMF.

JEL: $J 7 O$.

\section{Introdução}

O processo de integração da economia brasileira, no cenário da globalização mundial, promoveu a reconfiguração do processo produtivo e inúmeras consequências para o mercado de trabalho. No que concerne às reconfigurações da produção, ela passou da ótica da indústria para os serviços, o que implicou na criação de postos de trabalho mais vulneráveis a riscos e as tendências macroeconômicas nacionais (RAMOS, 2002). Nesse cenário, surgem novas formas de contratação, além do acentuado desemprego nos anos de 1990 (POCHMANN, 1998; 1999; DEDECCA, 1998; BALTAR, 2003).

No contexto de reestruturação produtiva, paralelamente ao avanço da economia brasileira na competitividade internacional, a força de trabalho foi utilizada como ajuste à tendência global da produção. A desregulamentação do mercado de trabalho e ações do mercado - no livre jogo entre contratador e contratado foram evidentes na economia do país (JATOBÁ e ANDRADE, 1993). Assim, a década de 1990 consagra-se por elevadas taxas de desemprego e forte incidência de trabalho precário nunca visto no país. 
Os problemas estruturais assim como os institucionais ${ }^{1}$ permitiram o surgimento de novas formas de contratação: em tempo parcial ou contratos sem registro em carteira. Por seu turno, a dinâmica estrutural da produção foi marcada pela ausência de uma política de desenvolvimento regional, em âmbito nacional, ocasionando a crescente disputa territorial por investimentos via incentivos fiscais.

Essa conjuntura brasileira permitiu o processo de desindustrialização das regiões mais ricas do país e a ampliação do parque industrial de unidades mais fragilizadas. Conforme Cano (1997), Diniz (1995) e Pacheco (1999), esse processo ocorreu com maior intensidade entre 1970-1985 e num menor ritmo pós 1985. Todavia, os postos de trabalhos criados nesse processo destacam-se por serem, em boa medida, terceirizados, mal remunerados e de alta rotatividade.

As regiões e estados beneficiados com a industrialização via incentivos fiscais gozaram da elevação no número de unidades fabris e na geração de empregos. Entretanto, não foi possível ficar incólume ao padrão de empregos gerados. Em regiões mais pobres, a exemplo do Nordeste brasileiro, os postos de trabalhos mostraram-se, sobremaneira, mais precários que os observados em regiões de maior envergadura, em sua capacidade produtiva e em sua estrutura industrial (SILVA FILHO e QUEIROZ, 2011). Além disso, o desemprego aberto metropolitano foi registrado com maior veemência nas regiões fragilizadas, além da incidência do desemprego oculto pelo desalento e pelo trabalho precário (BASTOS, 2010).

No tocante ao desemprego, estudos empíricos têm mostrado elevada concentração em grupos sociais mais vulneráveis, assim como em regiões mais propícias a esse fenômeno, tanto em escala internacional (OSÓRIO DE ALMEIDA et al,1995; FUNKHOUSER, 1996) quanto em âmbito nacional (BARROS, et al 1997; CORSEUIL et al, 1999; BASTOS, 2010). Em escala regional, Silva Filho (2011) constatou forte incidência do fenômeno concentrado, em sua grande maioria, em mulheres, jovens e não brancos, nas áreas metropolitanas de Fortaleza, Recife e Salvador. Além disso, nessas mesmas regiões metropolitanas foram registrados elevados índices de empregos informais ao longo dos anos 2000.

Ante isso, é pretensão deste artigo observar o mercado de trabalho no Ceará e na Região Metropolitana de Fortaleza, destacando-se, sobremaneira, o desemprego e o perfil do desempregado metropolitano e de todo o Estado. Para tanto, utilizam-se dados da Pesquisa Nacional por Amostra de Domicílios - PNAD, para os anos de 2001-2008. Quanto aos procedimentos metodológicos, obsrevam-se as caracteísticas determinantes do desemprego na área de abrangência da pesquisa, a partir do método utilizado por Corseuil et al, (1997), aplicado por Bastos (2010).

1 Define-se como a perda do poder de barganha do Estado e dos órgãos de defesa do trabalhador, em atuar em prol da força de trabalho, numa economia desregulada e de livre concorrência. 
Para atingir os objetivos propostos pelo estudo, o artigo está assim estruturado: além destas considerações iniciais, a segunda seção apresenta uma breve revisão de literatura acerca do desemprego e da vulnerabilidade de alguns grupos sociais na América Latina e no Brasil; a terceira seção reporta-se ao mercado de trabalho cearense e a sua estrutura; a quarta seção faz a análise descritiva dos dados para o estado do Ceará e da RMF; em seguida, a quinta seção apresenta os procedimentos metodológicos utilizados para observar a composição do desemprego; na sexta analisam-se os resultados da pesquisa e, por último, chega-se às considerações finais.

\section{Desemprego e vulnerabilidade no mercado de trabalho: algumas considerações ${ }^{2}$}

Analisar o desemprego nas economias em desenvolvimento sugere, sempre, a retomada do discurso acerca da reestruturação produtiva e dos seus impactos sobre o mercado de trabalho na periferia do capitalismo mundial ${ }^{3}$. Diante disso, com o avanço do capitalismo global, guiado pela busca de novos mercados e por condições de produção pautadas por baixos custos, além do avanço da ideologia de Estado mínimo e das ações do sistema neoliberal, o mercado de trabalho é massificado pela livre ação entre oferta e demanda, sendo o preço da mão-de-obra, em muitos casos, fator determinante para atração de recursos de capital.

Desse modo, com a reestruturação baseada na livre mobilidade de capitais, com o avanço da tecnologia de ponta, desencadeada pela robótica e pela microinformática, as economias menos desenvolvidas são afetadas, tanto na concorrência por mercados quanto na desocupação da força de trabalho mais vulnerável. Essas transformações radicais nos paradigmas da produção foram necessárias para ganhos de escala e de escopo e para ampliação do sistema capitalista pelo mundo. No entanto, cabe destacar que os problemas para o mercado de trabalho foram de grande intensidade, surgindo com isso, novas formas de contratação, redução de empregos com carteiras e contratação por hora.

O papel do Estado regulador perdeu espaço na dimensão da livre mobilidade de capitais e dos mercados em livre concorrência. Os resultados mais catastró-

2 O Ministério do Trabalho e do Emprego chama atenção para o cuidado que se deve ter ao qualificar o conceito de vulnerabilidade no mundo do trabalho. Isto porque, em geral, o que se observa em inúmeros estudos é a pouca ou nenhuma discussão do seu significado conceitual. Nesse sentido, o MTE (2007), interpreta/entende que "as situações de vulnerabilidade no mercado de trabalho [são] determinadas pela situação desvantajosa de grupos ou indivíduos em relação à posse desses ativos/atributos (físicos, pessoais e sociais), [e] podem ainda ser agravadas pelas superposições possíveis das diversas dimensões de vulnerabilidade. Neste sentido, é necessário que as situações de vulnerabilidade sejam expressas por indicadores capazes de captar essas superposições e também de revelar como ou em que intensidade cada uma dessas dimensões afetam a situação de vulnerabilidade no mundo do trabalho". (MTE-DIEESE, 2007, p.26-27).

3 Não é pretensão deste estudo entrar na imensidão da seara da globalização e da reestruturação produtiva, apenas se faz menção para os possíveis impactos na qualidade dos postos de trabalho e do desemprego estrutural, por ela causado. 
ficos dessas ações foram observados no mercado de trabalho e, sobremaneira, nos grupos sociais mais vulneráveis a tais ações. Tanto o emprego precário quanto o desemprego aberto em escala elevada assolaram as economias menos desenvolvidas e os grupos étnicos menos protegidos em países em desenvolvimento. O resultado da ausência do estado, da perda de barganha do poder sindical e da desregulamentação das leis do trabalho foi sentido, sobretudo, nos períodos de crescimento econômico crítico.

Diante disso, em todo o mundo em desenvolvimento, assistiu-se à elevação dos contratos informais de trabalho e consequentemente à redução dos postos com registros em carteira de trabalho. Paralelamente, o desemprego assolou essas economias e acentuou problemas de ordem sociais já elevados. Funkhouser (1996) e Marcoullier et al (1997) observaram que a informalidade no mercado de trabalho tem maior dimensão para a População Economicamente Ativa feminina, não branca, idosa e muito jovem. Essas características resultam da seletividade do mercado de trabalho e do crescimento na oferta de emprego inferior ao crescimento da PEA desses países.

Pela mesma ótica, Márquez e Pages (1998), Freije (2001) e Corbacho (2002) acompanharam o mercado de trabalho em países latino-americanos e constataram que, além do sexo, a idade da PEA é determinante na busca e manutenção de uma vaga no mercado de trabalho. A mão-de-obra feminina, conforme os autores, têm maiores possibilidades no mercado informal de trabalho, além disso, elas são mais expostas ao desemprego.

Essas características são, portanto, determinantes do desemprego em países em desenvolvimento. Ozório de Almeida et al (1995) observou em países latino-americanos, que a raça/cor é requisito que influencia a consecução vaga no mercado de trabalho, sendo, pois, os não brancos maioria relativa no contingente populacional de desempregados, além de serem eles maioria relativa no estoque de empregados na informalidade. Trata-se, porém, de um mercado de trabalho excludente e seletista, onde a força de trabalho é massificada diante de características étnicas, caso em que o desemprego e o subemprego afetam, em maior escala, os grupos sociais já citados.

Hirata (2009) observou que as mulheres são minorias nos postos de trabalhos de maior projeção social, além de encontrarem maiores dificuldades em ingressar no mercado de trabalho formal. Os estudos empíricos de Corseuil et al (1997), Corseuil (1999) e Bastos (2010), dentre outros, também para o Brasil, demonstram a incidência do desemprego, sobremaneira para idosos, jovens e mulheres, além da localização regional de a PEA determinar o desemprego e o seu tempo de permanência. Corroborando tais achados, Bastos (2010) constata elevadas taxas de desemprego aberto, desemprego oculto pelo desalento e desemprego oculto pelo trabalho precário, com maior incidência nos grupos étnicos e etários anteriormente citados.

Conforme Barros et al (1997), cabe ainda destacar a diferença gritante do 
desemprego entre os grupos sociais no Brasil, o que em seu contexto histórico já era evidenciada. Para o autor, mesmo que o histórico brasileiro de desemprego tenha se mantido com registros de baixas taxas até o início dos anos de $1990^{4}$, é possível que, mesmo nesse período, as taxas de desemprego agregadas tenham ocultado o problema do desemprego por grupos sociais, que divergem acentuadamente nas economias em desenvolvimento.

No caso do Nordeste metropolitano, estudos recentes ${ }^{5}$ têm demonstrado elevados índices de empregos informais, que atingem principalmente mulheres, não brancos, jovens e idosos, além das elevadas taxas de desemprego aberto, que atingem, sobremaneira, os grupos sociais já citados. Ante isso, percebe-se que os estudos empíricos, para as economias em desenvolvimento, convergem no que concerne ao desemprego. Há, portanto, discriminação e segregação no mercado de trabalho, e a população mais vulnerável tem as mesmas características sócio-econômicas e demográficas, notando-se ainda que, a diferenciação regional acentua ainda mais as diferenças entre aqueles que estão ou que experimentam constantemente o desemprego.

\section{Ceará: vulnerabilidade no mercado de trabalho, desemprego e emprego}

As transformações estruturais da produção e do trabalho são responsáveis pela nova dinâmica do mercado de trabalho brasileiro. Esse fenômeno de precarização do trabalho e de segregação e discriminação do trabalhador vem afetando sobremaneira as economias em desenvolvimento. No caso brasileiro, estudos empíricos têm demonstrado a intensidade desse fenômeno, além de evidenciar o desemprego diferencial por região do país (CORSEUIL et al, 1999). O Nordeste apresenta, sobremaneira, elevado diferencial tanto de estrutura produtiva quanto da performance ocupacional da força de trabalho.

O estado do Ceará não ficou incólume ao processo de segregação e discriminação no mercado de trabalho, acentuando as diferenças de rendimentos por raça/cor e sexo (MIRO e SULIANO, 2010; APARICIO e QUEIROZ, 2011), além de aglomerar maior contingente de desempregados nas mesmas características e somar ainda a questão da idade (SILVA FILHO, 2011; APARICIO e QUEIROZ, 2011). Além disso, no Ceará, é possível observar o diferencial de rendimento médio nos mesmos segmentos, comparando-se a RMF e o interior do estado. Silva Filho e Queiroz (2009) observaram

4 Corseuil et al (1999) propõe que a economia brasileira é caracterizada em seu percurso histórico com baixas taxas de desemprego, além da baixa produtividade marginal do trabalho (até década de 1980). Nesse caso, a reestruturação produtiva pode ter elevado o desemprego, dado, sobretudo, pela elevação da produtividade marginal do trabalho, somando-se a inovação tecnológica utilizadas no processo de produção. Com isso, assistiram-se nos anos de 1990, as mais elevadas taxas de desemprego aberto já registradas no Brasil.

5 Ver, por exemplo, Silva Filho (2011) e Aparicio e Queiroz (2011). 
que, em 2006, enquanto somente 7,28\% dos ocupados na indústria formal na $\mathrm{RMF}$ recebiam rendimento de até 1 salário mínimo, no restante do estado esse percentual era de $14,16 \%$. Somando-se a isso $82,90 \%$ na RMF e 90,88\% no interior do estado, o rendimento chegava até 2 salários mínimos no setor em questão. Com esses dados, percebe-se a diferença regional dentro da mesma unidade da federação, como também o baixo rendimento médio do trabalho no segmento que, além de ser formal é, em tese, o que melhor remunera, ou remunerava em anos pretéritos em todo o país.

Chama atenção a baixa taxa de formalidade nos postos de trabalho na RMF na comparação com outras RMs do Nordeste. Silva Filho (2011), utilizando dados da PNAD para o período de 2001-2008, observou que, embora a RMF tenha a menor taxa de desemprego, se comparada à Região Metropolitana de Recife e Salvador, nela concentra-se a maior taxa de informalidade, bem como o menor rendimento médio entre os ocupados nas categorias selecionadas para o estudo ${ }^{6}$. Além disso, há diferenças entre a formalidade e informalidade, considerando-se sexo, raça/cor e idade da PEA, principalmente entre o metropolitano e o interior do Ceará. No caso específico do estado do Ceará, conforme a Figura 1, que apresenta a população ocupada por condição de proteção no trabalho no Ceará e na $\mathrm{RMF}$, constata-se que entre os anos de 2001 a 2008 houve redução das taxas de desemprego tanto metropolitano quanto de todo o estado. Além do mais, reduziu-se, embora levemente, a participação dos ocupados sem carteira em detrimento daqueles com contratos formais de trabalho.

Quando se observa a formalidade dos postos de trabalho é evidente a menor taxa de participação no Estado, em detrimento da RMF. Em 2001, quando a PNAD foi a campo, encontrou apenas $23,1 \%$ de ocupados protegidos no estado do Ceará, enquanto esse percentual se elevava para 39,6\% na RMF. Em 2008, o percentual de protegidos no trabalho eleva-se para $28,4 \%$ no âmbito estadual e para 43,2\% no tecido metropolitano. Vale frisar que o crescimento de 5,3\% na população ocupada e protegida no estado e de $3,6 \%$ na RMF no período em análise, pode estar relacionado à interiorização das atividades produtivas via políticas de interiorização das indústrias cearenses, a partir das ações do Fundo de Desenvolvimento Industrial do Ceará - FDI . Por seu turno, a dinâmica econômica metropolitana, com maior concentração de ocupações estatutárias, militares e industriais, por assim dizer, permite observar maior percentual de proteção no trabalho na RMF, em comparação às áreas interioranas do estado ${ }^{8}$.

6 Ver Silva Filho (2011), Mercado de trabalho e estrutura sócio-ocupacional: estudo comparativo entre as regiões metropolitanas de Fortaleza, Recife e Salvador - 2001-2008. (Dissertação de Mestrado em Economia) <http: www.observatoriodasmetropoles.net/download/dissertacao_luis_abel.pdf $>$.

7 Para melhor compreensão ver Pontes, Vianna e Holanda (2006); Silva Filho e Queiroz (2009b)

$8 \mathrm{Na}$ figura 1, considera-se formal os trabalhadores com carteira de trabalho assinada, além dos empregadores com previdência e dos militares e estatutários. 
FIGURA 1: POPULAÇÃO OCUPADA POR CONDIÇÃO DE PROTEÇÃO NO TRABALHO (\%) - CEARÁ E RMF - 2001-2008

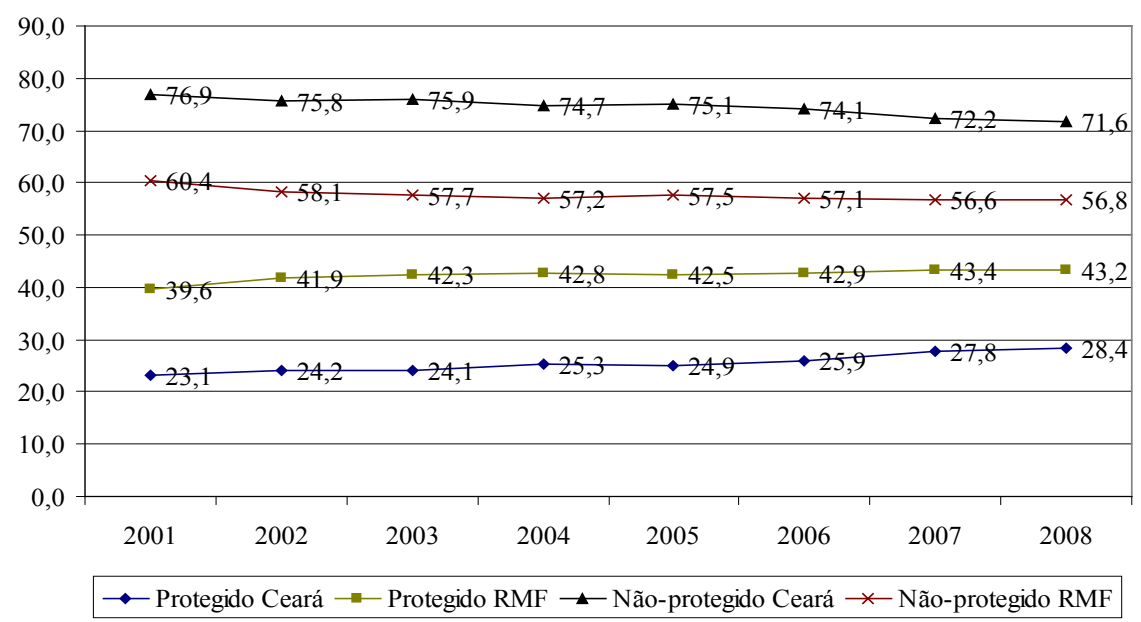

Fonte: PNAD, 2001 - 2008 / IBGE

Ainda na Figura 1, é possível observar a leve tendência que segue o mercado de trabalho em reduzir a participação dos ocupados sem carteira e elevar a participação dos contratos formais de trabalho tanto em nível estadual quanto metropolitano. Sem dúvida, a melhoria nos indicadores econômicos do país, observadas a partir de 2004, tem sido responsável pela criação de postos de trabalho com vínculos formais (REMY, QUEIROZ e SILVA FILHO, 2010). Nesse contexto, o Ceará tem acompanhado a tendência observada em nível nacional e regional, embora seu crescimento relativo, na criação de postos formais de trabalho, seja inferior ao observado em unidades com padrões de produção semelhantes ${ }^{9}$.

Essa seção apresentou algumas considerações acerca do mercado de trabalho cearense, com ênfase no estado e na RMF. Levaram-se em consideração diferenças regionais e abordaram-se alguns achados que relatam a discriminação por sexo, raça/cor e idade da PEA. A seção que segue aborda o foco central desse estudo - o desemprego no Ceará e na RMF, para em seguida apresentar os seus determinantes.

\section{Estatísticas do desemprego recente no Ceará e na Região Metropolitana de Fortaleza: 2001-2008}

O desemprego no estado do Ceará tem dinâmica acoplada às variações macroeconômicas nacionais. Na Figura 2, observa-se que o ano de maior incidência de desemprego no estado e na RMF foi 2003 Justamente 9 Nesse caso, refere-se aos estados da Bahia e de Pernambuco. 
nesse ano, o Brasil registrou a menor taxa de crescimento do Produto Interno Bruto (1,1\%) na década de 2000 (BASTOS, 2010), e o Ceará também apresentou baixo crescimento (1,4\%) no seu PIB (IPECE, 2011).

Por outro lado, a partir de 2004, verifica-se tendência de redução nas taxas de desemprego no estado e na RMF. Porém, constata-se que o desemprego metropolitano ao longo da série estudada, sempre foi superior ao do estado.

Durante os anos observados, o desemprego aberto no Ceará foi sempre de um dígito, diferentemente da RMF, que, somente no ano de 2008 alcançou tal resultado (FIGURA 2). Nesse último ano, a PNAD encontrou apenas 6,2\% da PEA do estado desempregada e 8,7\% na RMF. Cabe relatar que houve redução do desemprego aberto na área de estudo quando comparado o primeiro ao último ano da série. A RMF reduz de 11,9\% em 2001 para 8,7\% em 2008 o percentual de desempregados. Já o estado passa de 7,1\% para 6,2\% entre 2001 e 2008, respectivamente.

FIGURA 2: EVOLUÇÃO DO DESEMPREGO (\%) - CEARÁ E RMF - 2001-2008

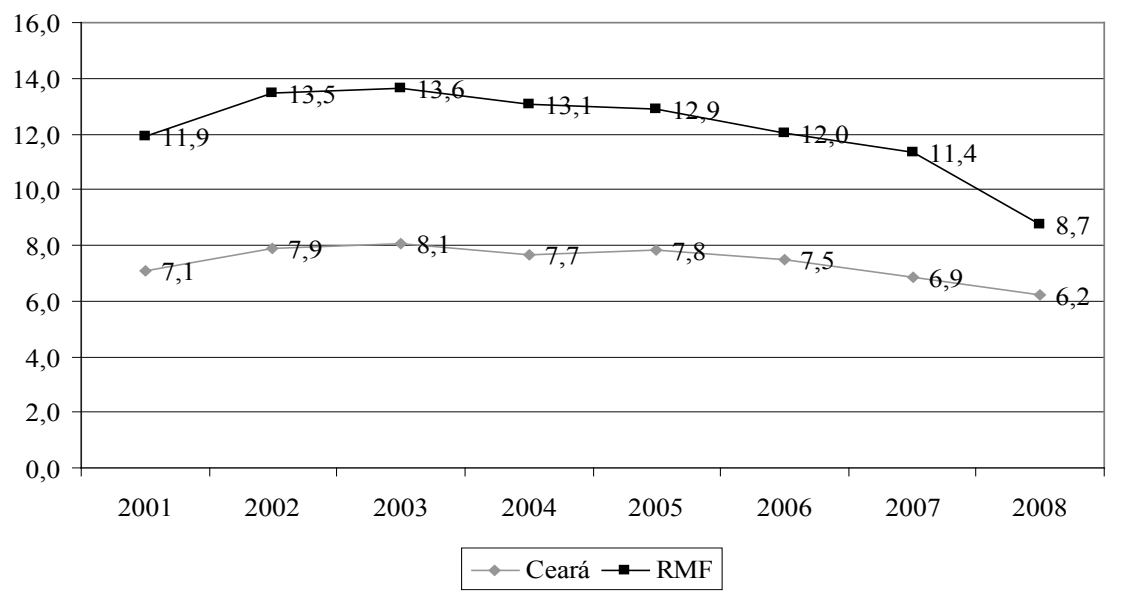

Fonte: PNAD, 2001 - 2008 / IBGE

Quando se analisa o desemprego segundo o sexo, os dados da Figura 3 revelam as maiores taxas para as mulheres na RMF e as menores para os homens em todo o estado. É possível, portanto, observar que ocorreu redução das taxas de desemprego do primeiro para o último ano analisado para ambos os sexos e em ambas as áreas estudadas. Em 2001, enquanto a taxa de desemprego masculina no estado do Ceará era de 6,2\%, na RMF o desemprego atingia 10,4\% da população do mesmo sexo. Ainda em 2001, o desemprego feminino foi superior ao masculino em todo o estado $(8,2 \%)$ e inferior ao feminino na $\mathrm{RMF}(13,8 \%)$. 
FIGURA 3: DESEMPREGADOS SEGUNDO O SEXO (\%) - CEARÁ E REGIÃO METROPOLITANA DE FORTALEZA - 2001-2008

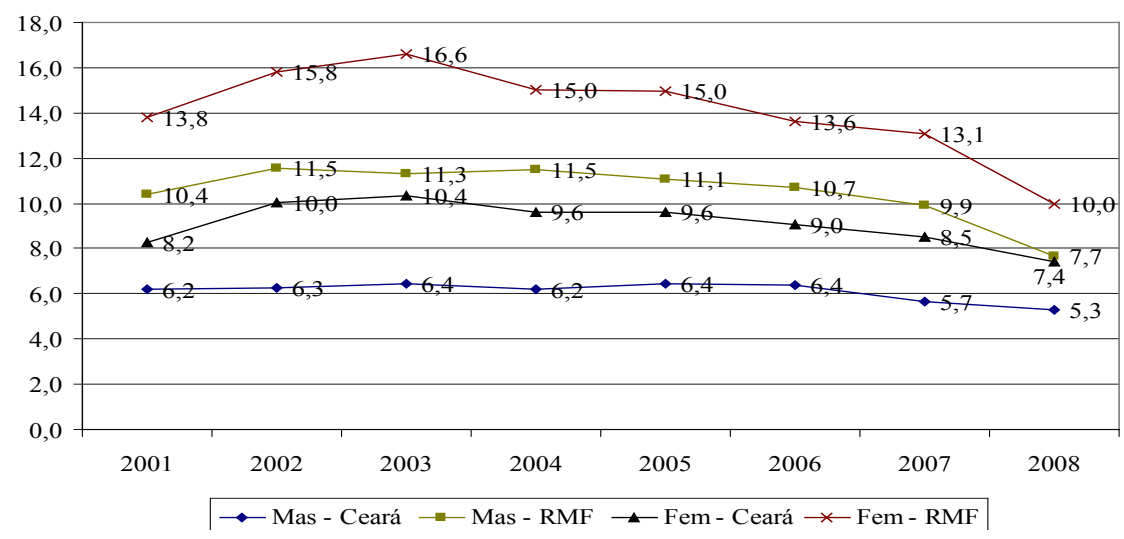

Fonte: PNAD, $2001-2008$ / IBGE

O comportamento das taxas de desemprego permaneceu com leves alterações ao longo dos oito anos em observação, reduzindo-se levemente, para o sexo masculino e feminino, tanto na UF quanto na Região Metropolitana. O desemprego metropolitano feminino, que atingiu 13,8\% dessa PEA em 2001, reduz-se para 10,0\% em 2008, enquanto para todo o estado, essa redução foi de 8,2 para $7,4 \%$. Para a PEA masculina, o desemprego no estado caiu de $6,2 \%$ para 5,3\% e, na $\mathrm{RMF}$, de 10,4\% para 7,7\%, em 2001 e 2008, respectivamente.

Quando se observa o desemprego segundo a raça/cor, os dados mostram redução das taxas de desempregos tanto para brancos quanto para não brancos, em ambas as áreas analisadas, entre 2001 a 2008. Contudo, cabe destacar que, ao comparar o percentual de desempregados para o Ceará, no primeiro ano, a PEA branca era de $6,4 \%$ e de $7,4 \%$ para a PEA não branca. Na RMF o percentual de desocupados segundo as mesmas características demográficas eram de 10,3\% para o primeiro grupo e 13,1\% para o segundo em 2001.

Na Região Metropolitana o maior índice de desemprego foi registrado para o grupo de não brancos em 2003 (14,8\%), já para os brancos a maior taxa registrada ocorreu no ano de 2002 (8,2\%), não sendo superior a um dígito em nenhum ano. No caso do estado, os dados revelam o pior desempenho do mercado de trabalho para a PEA de cor branca em $2003(7,7 \%)$ e para a não branca $(8,2 \%)$ no mesmo ano.

Entre 2001 e 2008, os dados mostram redução de 13,1\% para 8,6\% no percentual de não brancos desempregados na RMF, sendo esse o movimento mais acentuado; e, de 10,3\% para 8,5\%, a redução do percentual de desempregados brancos na mesma região. No estado como um todo, o desemprego da PEA não branca baixou de $7,4 \%$ para $6,3 \%$, do primeiro para o último ano, bem como de $6,4 \%$ para 5,7\% da PEA que se declararam brancos à PNAD nos mesmos anos. 
FIGURA 4: DESEMPREGADOS SEGUNDO RAÇA/COR (\%) - CEARÁ E REGIÃO METROPOLITANA DE FORTALEZA - 2001-2008

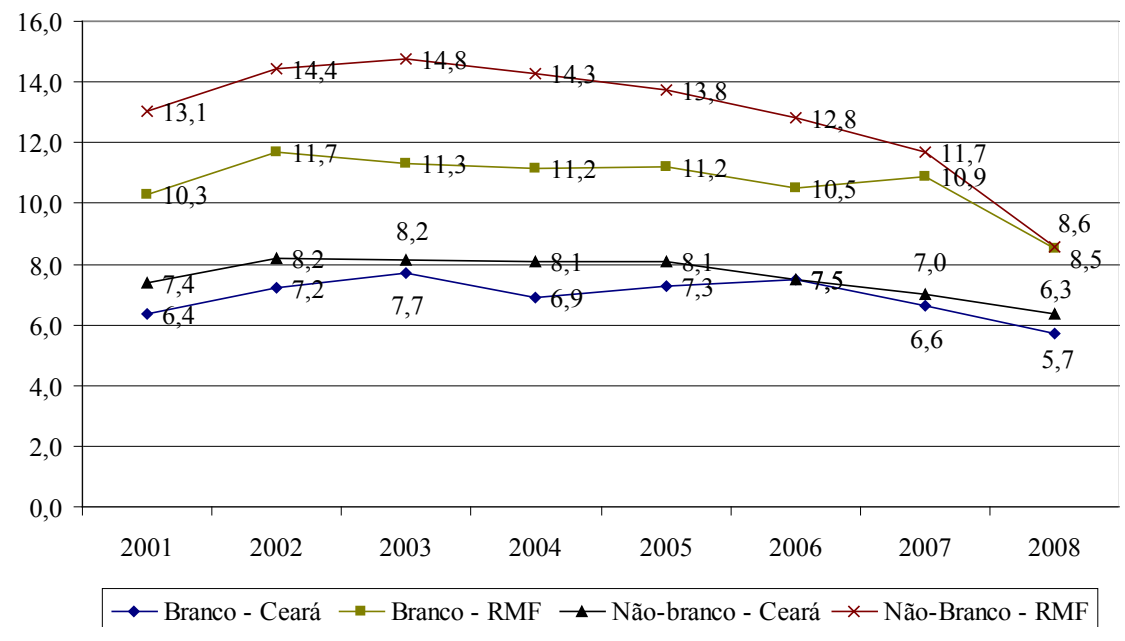

Fonte: PNAD, 2001 - 2008 / IBGE

Para se observar o nível que atingiu o desemprego no Ceará e na RMF, segundo a idade da PEA, os dados plotados na Tabela 1 mostram que, em todas as faixas etárias e em todos os anos observados, o desemprego metropolitano foi superior ao de todo o estado, indo de encontro à teoria.

As maiores taxas de desemprego estadual e metropolitano incidiram sobre a PEA com idade entre 15 e 24 anos em todos os anos observados. O desemprego metropolitano chegou a atingir 27,0\% deles na RMF, em 2004. Em todo o estado, a maior taxa foi de 16,5\% em 2006. Todavia, para essa faixa etária, os dados ratificam o encontrado na literatura para países latino-americanos (MÁRQUEZ e PAGES, 1998; FREIJE, 2001; e, CORBACHO, 2002) e para o Brasil (BARROS et al (1997); BASTOS, 2010), sendo, portanto, convergentes os resultados encontrados nesse estudo aos demais aqui citados.

Há, todavia, a necessidade de se considerar a trajetória diferenciada das taxas de desemprego segundo a idade da PEA. No estado do Ceará, elevou-se o desemprego para aqueles com idade entre 10 e 14 anos, movimento diferente do observado na RMF que, contrariamente, reduziu o percentual quando comparado o primeiro ao último ano. Para as demais faixas etárias, registrou-se redução do desemprego tanto em nível estadual quanto metropolitano, entre 2001 e 2008. 
TABELA 1: DESEMPREGADOS SEGUNDO FAIXA ETÁRIA(\%) - CEARÁ E REGIÃO METROPOLITANA DE FORTALEZA - 2001-2008

\begin{tabular}{c|ccc|c|c|c|c|c|c|c|c}
\hline \multirow{2}{*}{ Ano } & \multicolumn{3}{|c|}{ De 10 a 14 } & \multicolumn{3}{c|}{ De 15 a 24 } & \multicolumn{2}{c|}{ De 25 a 39 } & \multicolumn{2}{c|}{ De 40 a 59 } & \multicolumn{2}{|c}{$\begin{array}{c}\text { anos ou } \\
\text { mais }\end{array}$} \\
\cline { 2 - 12 } & Ceará & RMF & Ceará & RMF & Ceará & RMF & Ceará & RMF & Ceará & RMF \\
\hline 2001 & 2,7 & 8,6 & 14,6 & 22,7 & 6,4 & 9,9 & 3,5 & 6,4 & 0,9 & 4,4 \\
2002 & 5,0 & 12,6 & 15,8 & 26,2 & 7,2 & 11,2 & 3,4 & 6,1 & 0,7 & 2,5 \\
2003 & 3,6 & 14,1 & 15,9 & 26,0 & 7,4 & 11,5 & 3,9 & 6,8 & 1,5 & 4,6 \\
2004 & 3,0 & 6,0 & 15,4 & 27,0 & 6,8 & 10,7 & 3,8 & 6,2 & 1,2 & 3,4 \\
2005 & 4,1 & 12,7 & 16,4 & 26,7 & 6,9 & 10,9 & 3,9 & 5,9 & 0,8 & 1,4 \\
2006 & 2,6 & 11,1 & 16,5 & 25,6 & 6,9 & 9,8 & 3,2 & 5,7 & 0,8 & 2,3 \\
2007 & 3,4 & 15,9 & 14,1 & 23,1 & 6,6 & 9,9 & 3,0 & 5,6 & 1,6 & 2,7 \\
2008 & 4,6 & 7,9 & 13,7 & 19,8 & 5,9 & 7,2 & 2,4 & 3,8 & 1,2 & 1,6 \\
\hline
\end{tabular}

Fonte: PNAD, 2001 - 2008 / IBGE

Impõe-se, porém, destacar que, contrariando o amplamente difundido na literatura acerca do desemprego e os seus impactos segundo as faixas etárias, no estado do Ceará e na RMF, os menores índices de desemprego encontrados foram para a PEA com idade de 60 anos ou mais. Todavia, conforme Silva Filho (2011), 80,9\% dos ocupados nessa faixa etária estavam desprotegidos no trabalho no ano de 2008.

Quanto ao desemprego segundo os anos de estudo da PEA, os dados da Tabela 2 apontam para menores percentuais de desempregados, exatamente para aqueles com menos anos de estudo. Esse resultado é, portanto, condicionado por fatores inerentes ao mercado de trabalho local, além das condições de trabalhos aceitas por tais indivíduos. Para a população com escolaridade entre o e 4 anos de estudo, o desemprego afetava 3,9\% e 9,1\% no estado e na RMF, respectivamente, no ano de 2001, reduzindo-se para 2,8\% e 5,2\% em 2008, respectivamente. Por outro lado, são eles que ocupam os maiores percentuais de desprotegidos no trabalho, chegando a atingir 78,2\% em 2008, na RMF, sendo ainda o maior percentual de desprotegidos entre as RMs do Nordeste (SILVA FILHO, 2011).

Para aqueles com escolaridade entre 5 e 8 anos de estudo, observou-se redução das taxas de desemprego tanto no âmbito estadual quanto metropolitano, quando se compara 2001 a 2008. Para tanto, o desemprego na RMF atingiu mais de um dígito em quase todos os anos, à exceção de 2008 (8,7\%), sendo que somente em 2001 (10,2\%) esse percentual superou um dígito em todo o estado cearense. 
TABELA 2: DESEMPREGADOS SEGUNDO FAIXA DE ESCOLARIDADE (\%) - CEARÁ E REGIÃO METROPOLITANA DE FORTALEZA - 2001-2008

\begin{tabular}{|c|c|c|c|c|c|c|c|c|c|c|}
\hline \multirow[t]{2}{*}{ Ano } & \multicolumn{2}{|c|}{ De 10 a 14} & \multicolumn{2}{|c|}{ De 15 a 24} & \multicolumn{2}{|c|}{ De 25 a 39} & \multicolumn{2}{|c|}{ De 40 a 59} & \multicolumn{2}{|c|}{$\begin{array}{l}60 \text { anos ou } \\
\text { mais }\end{array}$} \\
\hline & Ceará & $\mathrm{RMF}$ & Ceará & $\mathrm{RMF}$ & Ceará & $\mathrm{RMF}$ & Ceará & $\mathrm{RMF}$ & Ceará & $\mathrm{RMF}$ \\
\hline 2001 & 2,7 & 8,6 & 14,6 & 22,7 & 6,4 & 9,9 & 3,5 & 6,4 & 0,9 & 4,4 \\
\hline 2002 & 5,0 & 12,6 & 15,8 & 26,2 & 7,2 & 11,2 & 3,4 & 6,1 & 0,7 & 2,5 \\
\hline 2003 & 3,6 & 14,1 & 15,9 & 26,0 & 7,4 & 11,5 & 3,9 & 6,8 & 1,5 & 4,6 \\
\hline 2004 & 3,0 & 6,0 & 15,4 & 27,0 & 6,8 & 10,7 & 3,8 & 6,2 & 1,2 & 3,4 \\
\hline 2005 & 4,1 & 12,7 & 16,4 & 26,7 & 6,9 & 10,9 & 3,9 & 5,9 & 0,8 & 1,4 \\
\hline 2006 & 2,6 & 11,1 & 16,5 & 25,6 & 6,9 & 9,8 & 3,2 & 5,7 & 0,8 & 2,3 \\
\hline 2007 & 3,4 & 15,9 & 14,1 & 23,1 & 6,6 & 9,9 & 3,0 & 5,6 & 1,6 & 2,7 \\
\hline 2008 & 4,6 & 7,9 & 13,7 & 19,8 & 5,9 & 7,2 & 2,4 & 3,8 & 1,2 & 1,6 \\
\hline
\end{tabular}

Fonte: PNAD, 2001 - 2008 / IBGE

A PEA com escolaridade entre 9 e 11 anos de estudo experimentou as maiores taxas de desemprego aberto no período analisado. Em todos os anos observados, o desemprego foi de dois dígitos tanto no estado quando na RMF. Seja pela busca de emprego com melhores condições de trabalho, seja pela não aceitação de empregos precários ou pela pouca oportunidade de empregos para mão-de-obra qualificada, o fato é que o desemprego foi substancialmente elevado exatamente para aqueles com nível de escolaridade considerável tanto no tecido metropolitano quanto em todo o estado.

Já a PEA com 12 anos ou mais de estudo experimentaram desemprego somente superior àqueles com os menores índices de escolaridade. No estado do Ceará, a taxa de desocupados saiu de 5,8\% em 2001, para 6,0\% em 2008, mostrando leve aumento do primeiro para o último ano analisado. Já na RMF, embora superior ao observado em todo o estado, o movimento foi contrário, caindo de 7,5\%, em 2001, para 7,2\%, em 2008.

\section{Procedimentos metodológicos}

Neste estudo utiliza-se o método proposto por Corseuil (1997) e replicado por Bastos (2010). Para tanto, a fonte de dados utilizada por Corseuil (1997) foi a Pesquisa Mensal de Emprego (PME) do Instituto Brasileiro de Geografia e Estatística - IBGE, enquanto Bastos (2010) recorreu aos dados da Pesquisa de Emprego e Desemprego (PED) do DIEESE/SEADE. Por seu turno, nessa investigação, a base usada para a composição do desemprego é calculada a partir da Pesquisa Nacional por Amostra de Domicílios (PNAD) do IBGE. 
A área de abrangência da pesquisa compreende o desemprego em todo o estado do Ceará e na Região Metropolitana de Fortaleza ${ }^{10}$. Parte-se do pressuposto de que o desemprego metropolitano é mais acentuado, seja pelo fato da aglomeração populacional em busca de maiores oportunidades de trabalho concentrarem-se nestas localidades ou pela existência de maiores possibilidades de emprego no tecido metropolitano brasileiro, que apresenta elevada concentração de atividades produtivas.

Para a construção da composição do desemprego, foram utilizadas as seguintes variáveis:

- Estoque total de desempregados: pessoas que se declararam desempregadas na semana de referência da pesquisa;

- Estoque de desempregados segundo o sexo: masculino e feminino;

- Estoque de desempregados segundo a raça/cor: branco e não branco, sendo que não brancos são compostos por pretos e pardos (amarelos e indígenas foram excluídos da amostra por sua baixa representatividade estatística);

- Estoque de desempregados segundo a faixa etária: 10 a 14; 15 a 24; 25 a 39; 40 a 59 ; 60 ou mais anos;

- Estoque de desempregados segundo os anos de estudo: o a 4; 5 a 8; 9 a 11; 12 ou mais anos.

A metodologia proposta por Corseuil et al (1997) investigou as metrópoles brasileiras, no período que compreende os anos de 1986 e 1995; e, posteriormente, Bastos (2010) replicou-a para as metrópoles brasileiras e para o Distrito Federal, na série temporal entre 1999 e 2007. Nesse estudo, além de outra fonte de dados utilizada pelos autores, o lócus de pesquisa é o estado do Ceará e a RMF.

Destarte, o desemprego de um grupo populacional de uma região pode ter a seguinte decomposição:

$$
\frac{U_{i}}{U}=\frac{U_{i}}{N_{i}} \cdot \frac{N_{i}}{P_{i}} \cdot \frac{P_{i}}{P} \cdot \frac{P}{N} \cdot \frac{N}{U}
$$

Onde:

$U$ = estoque de desempregados total no estado do Ceará e na RMF nos anos selecionados (2001-2008);

$U_{i}$ = assume o caráter de estoque de desempregados do grupo i no estado do Ceará e na RMF; tuba, Maranguape, Maracanaú, Eusébio, Guaiúba, Itaitinga, Chorozinho, Pacajus, Horizonte e São Gonçalo do Amarante. 
$N$ = número de membros da PEA total no estado do Ceará e na RMF;

$N_{i}=$ número de membros da PEA do grupo i tanto no estado quanto na RMF;

$P=$ representa o número de membros da PIA total no estado do Ceará e na RMF no período em análise.

$P_{i}=$ denominado como o número de membros da PIA do grupo i;

A partir da equação 1, reescreve-se a equação 2 da forma que se segue:

$$
\frac{U_{i}}{U}=\frac{U_{i} / N_{i}}{U / N} \cdot \frac{N_{i} / P_{i}}{N / P} \cdot \frac{P_{i}}{P}
$$

Transformando-se os dados da equação (2) em logaritmos naturais, pode-se captar o efeito elasticidade na decomposição dos fatores que afetam o desemprego.

Dessa forma, tem-se:

$$
\operatorname{Ln}\left(\frac{U_{i}}{U}\right)=\left[\operatorname{Ln}\left(\frac{U_{i}}{N_{i}}\right)-\operatorname{Ln}\left(\frac{U}{N}\right)\right]+\left[\operatorname{Ln}\left(\frac{N_{i}}{P_{i}}\right)-\operatorname{Ln}\left(\frac{N}{P}\right)\right]+\operatorname{Ln}\left(\frac{P_{i}}{P}\right)
$$

Com esta expressão temos as variações expressas da seguinte forma:

$$
\Delta \operatorname{Ln}\left(\frac{U_{i}}{U}\right)=\left[\Delta \operatorname{Ln}\left(\frac{U_{i}}{N_{i}}\right)-\Delta \operatorname{Ln}\left(\frac{U}{N}\right)\right]+\left[\Delta \operatorname{Ln}\left(\frac{N_{i}}{P_{i}}\right)-\Delta \operatorname{Ln}\left(\frac{N}{P}\right)\right]+\Delta \operatorname{Ln}\left(\frac{P_{i}}{P}\right)
$$

Apresentado o método, a expressão (4) propõe observar a variação da participação do grupo no estoque de desempregados de uma região. Interpretando Corseuil et al (1997) e Bastos (2010), a participação dos desempregados de um determinado grupo em uma determinada região está diretamente relacionada à variação do peso deste grupo populacional na PIA da região; a taxa de participação da força de trabalho do grupo na taxa da participação da força de trabalho da região; a participação da taxa de desempregos em relação a taxa total de desempregados existentes na região.

Diante dos procedimentos aqui adotados, os resultados da pesquisa estão expostos na seção que se segue.

\section{Evolução da participação no desemprego e os seus componentes no Ceará e na Região Metropolitana de Fortaleza - 2001 e 2008}

Diante dos dados aqui plotados, pode-se observar que houve redução da participação masculina no desemprego no estado do Ceará. Para tal resultado, todos os fatores colaboraram para tal declínio, com exceção da variação do peso do grupo da PIA do estado, que se elevou nos anos observados $(0,0104)$. 
Contrariamente ao verificado para os homens, no caso das mulheres, os resultados revelam elevação de sua participação no desemprego no estado. Para elas, o único fator que levemente contribuiu para retirá-las do desemprego foi a variação da PIA feminina, que em sentido oposto ao observado para os homens, diminuiu -0,0095 no período em foco. Já o comportamento da taxa de desemprego do grupo (0,0203), assim como o comportamento da taxa de participação da força de trabalho feminina no estado $(0,0339)$ elevaram-se, provocando o aumento da participação delas no desemprego em nível estadual.

TABELA 3: EVOLUÇÃO DA PARTICIPAÇÃO NO DESEMPREGO SEGUNDO O SEXO - CEARÁ E REGIÃO METROPOLITANA DE FORTALEZA - 2001 E 2008

\begin{tabular}{c|cc|cc}
\hline \multirow{2}{*}{ DESCRIÇÃO } & \multicolumn{2}{|c|}{ CEARÁ } & \multicolumn{2}{c}{ RMF } \\
\cline { 2 - 5 } & Masculino & Feminino & Maculino & Feminino \\
\hline$\Delta \ln \left(\frac{U_{i}}{U}\right)$ & $-0,0468$ & 0,0446 & 0,0058 & $-0,0054$ \\
$\Delta \ln \left(\frac{U_{i}}{N_{i}}\right)-\Delta \ln \left(\frac{U}{N}\right)$ & $-0,0281$ & 0,0203 & 0,0102 & $-0,0107$ \\
$\Delta \ln \left(\frac{N_{i}}{P_{i}}\right)-\Delta \ln \left(\frac{N}{P}\right)$ & $-0,0291$ & 0,0339 & $-0,0241$ & 0,0222 \\
$\Delta \ln \left(\frac{P_{i}}{P}\right)$ & 0,0104 & 0,0095 & 0,0197 & $-0,0169$ \\
\hline
\end{tabular}

Fonte: PNAD, 2001 - 2008 / IBGE.

Na RMF, como pode ser observado, ocorreu elevação da participação da força de trabalho masculina no estoque de desempregados. Nesse caso, tanto o comportamento da taxa de desemprego do grupo $(0,0102)$ em relação ao desemprego da região, quanto a variação do peso do grupo $(0,0197)$ na PIA na região corroboraram o resultado. Diante disso, somente o comportamento da taxa de participação da força de trabalho do grupo em relação à força de trabalho da região impactou positivamente no sentido de reduzir a participação deles no estoque total de desempregados $(-0,0241)$. Já para as mulheres, registrou-se redução da participação delas no estoque total de desempregados na RMF. Para tanto, o comportamento da taxa de desemprego feminino (-o,0107) em relação ao desemprego total, assim como a variação do peso do grupo $(-0,0169)$ na PIA da região responderam por tal efeito.

Em relação ao estoque de desempregados segundo a raça/cor, observou-se redução tanto de brancos quanto de não brancos, tanto no Ceará quanto na RMF, variando apenas a intensidade. Mas a redução da PEA branca $(-0,0324)$ no estoque total de desempregados no estado foi significativamente superior ao observado para os não brancos (-0,0062). No caso do primeiro, somente a variação do peso do grupo na PIA da região corroborou tal resultado, o 
que foi significativo, já que tanto o comportamento da taxa de desemprego do grupo em comparação com a taxa de desemprego do estado quanto o comportamento da taxa de participação da força de trabalho do grupo em comparação com o da taxa de participação na força de trabalho no estado contribuíram em sentido contrário, ou seja, para elevá-los no desemprego. Já para o segundo grupo, somente a variação do peso do grupo na PIA da região agiu em sentido contrário.

TABELA 4: EVOLUÇÃO DA PARTICIPAÇÃO NO DESEMPREGO SEGUNDO A RAÇA/COR - CEARÁ E REGIÃO METROPOLITANA DE FORTALEZA - 2001 E 2008

\begin{tabular}{c|c|c|c|c}
\hline \multirow{2}{*}{ DESCRIÇÃO } & \multicolumn{2}{|c|}{ CEARÁ } & \multicolumn{2}{c}{ RMF } \\
\cline { 2 - 5 }$\Delta \ln \left(\frac{U_{i}}{U}\right)$ & Branco & não branco & Branco & não branco \\
\hline \multicolumn{1}{c}{$-0,0324$} & $-0,0062$ & $-0,0633$ & $-0,0108$ \\
$\ln \left(\frac{U_{i}}{N_{i}}\right)-\Delta \ln \left(\frac{U}{N}\right)$ & 0,0194 & $-0,0265$ & 0,1246 & $-0,1058$ \\
$\Delta \ln \left(\frac{N_{i}}{P_{i}}\right)-\Delta \ln \left(\frac{N}{P}\right)$ & 0,0100 & $-0,0058$ & 0,0068 & $-0,0182$ \\
$\Delta \ln \left(\frac{P_{i}}{P}\right)$ & $-0,0618$ & 0,0260 & $-0,1947$ & 0,1132 \\
\hline
\end{tabular}

Fonte: PNAD, 2001 - 2008 / IBGE

Nota 1: Não branco corresponde à pretos e pardos.

Na RMF, somente a variação do peso do grupo de brancos na PIA da região contribuiu para a redução deles no estoque de desempregados, sendo que os demais fatores movimentaram de forma a elevar o desemprego. Já para os não brancos, tanto o comportamento da taxa de desemprego do grupo em relação ao desemprego total, bem como o comportamento da taxa de participação da força de trabalho do grupo em comparação com o da taxa de participação da força de trabalho da região colaboraram para a redução dos não brancos no estoque total de desempregados.

Quanto à composição do estoque de desempregados por faixa etária, os dados da Tabela 5 mostram que, para o primeiro grupo etário (10 a 14 anos), no estado do Ceará, se elevou a participação relativa deles no estoque de desempregado. Isto porque aumentou significativamente o comportamento da taxa de desemprego do grupo em relação à taxa de desemprego da região e, nesse caso, mesmo que os dois outros fatores tenham colaborado em declinação, tal fato não foi o suficiente.

Já na RMF, a participação no estoque total de desempregado, nessa faixa etária, apresentou redução. Para tal, tanto o comportamento da taxa de 
participação da força de trabalho do grupo em relação à força de trabalho da região, bem como a variação do peso deles na PIA da região variando negativamente fizeram reduzir relativamente o estoque total de desempregados.

TABELA 5: EVOLUÇÃO DA PARTICIPAÇÃO NO DESEMPREGO SEGUNDO A FAIXA ETÁRIA - CEARÁ E REGIÃO METROPOLITANA DE FORTALEZA - 2001 E 2008

\begin{tabular}{|c|c|c|c|c|c|c|c|c|c|c|}
\hline \multirow[t]{2}{*}{ Descrição } & \multicolumn{2}{|c|}{ De 10 a 14} & \multicolumn{2}{|c|}{ De 15 a 24} & \multicolumn{2}{|c|}{ De 25 a 39} & \multicolumn{2}{|c|}{ De 40 a 59} & \multicolumn{2}{|c|}{$\begin{array}{c}\text { Mais de } 60 \\
\text { anos }\end{array}$} \\
\hline & Ceará & RMF & Ceará & RMF & Ceará & RMF & Ceará & $\mathrm{RMF}$ & Ceará & RMF \\
\hline$\Delta \ln \left(\frac{U_{i}}{U}\right)$ & 0,090 & $-0,539$ & 0,001 & 0,082 & $-0,003$ & $-0,081$ & $-0,027$ & $-0,004$ & 0,172 & $-0,538$ \\
\hline$\Delta \ln \left(\frac{U_{i}}{N_{i}}\right)-\Delta \ln \left(\frac{U}{N}\right)$ & 0,287 & 0,219 & 0,027 & 0,179 & 0,021 & $-0,004$ & $-0,098$ & $-0,202$ & 0,156 & $-0,722$ \\
\hline$\Delta \ln \left(\frac{N_{i}}{P_{i}}\right)-\Delta \ln \left(\frac{N}{P}\right)$ & $-0,131$ & $-0,631$ & $-0,002$ & $-0,011$ & 0,000 & 0,020 & 0,004 & 0,004 & $-0,029$ & -0,019 \\
\hline$\Delta \ln \left(\frac{P i}{P}\right)$ & $-0,065$ & $-0,127$ & $-0,024$ & $-0,087$ & $-0,023$ & $-0,097$ & 0,068 & 0,193 & 0,045 & 0,203 \\
\hline
\end{tabular}

Fonte: PNAD, 2001 - 2008 / IBGE

No caso da faixa etária que compreende a PEA desocupada com idade entre 15 e 24 anos, observa-se elevação deles no estoque total de desempregados tanto no estado quanto na RMF. Em todo o estado, a elevação deles ocorreu em menor intensidade (o,o01) do que o observado na RMF (o,o82). Além disso, não só em âmbito estadual como também metropolitano, apenas o comportamento da taxa de participação da força de trabalho do grupo em comparação com o da taxa de participação na força de trabalho na região foi suficiente para elevar o estoque deles no desemprego.

Por outro lado, constatou-se redução do desemprego da PEA para o grupo de idade entre 25 a 39 anos no estoque total de desempregados tanto metropolitano quanto estadual. Nesse último, somente a variação do peso do grupo na PIA da região concorreu para tal resultado. Mas na RMF responderam por esse resultado tanto o fator citado quanto o comportamento da taxa de desemprego do grupo em comparação ao da taxa de desemprego da região.

Para a PEA desocupada com idade entre 40 e 59 anos assiste-se à redução da participação deles no estoque total de desempregados ao longo dos anos observados tanto no estado (-0,027) quanto na região metropolitana (-0,004). Para tanto, apenas o comportamento da taxa de desemprego do grupo em comparação ao da taxa de desemprego da região teve referência. Já na faixa etária que compreende a PEA desempregada com idade de 60 anos ou mais, no estado do Ceará verificou-se a elevação da participação deles no estoque total de desempregados $(0,172)$, diferentemente da RMF em que ocorreu 
movimento contrário $(-0,538)$. No Ceará, somente o comportamento da taxa de participação na força de trabalho do grupo em comparação ao da força de trabalho da região variou de forma a reduzir o estoque de desempregados, o que não foi suficiente para barrar tal fenômeno. Já na RMF, somente a elevação do peso do grupo na PIA da região contribuiu para elevá-los ao estoque total de desempregados, não sendo suficiente para promover a elevação dessa PEA no estoque total de desempregados.

Quando se observa a PEA desocupada, segundo os anos de estudo, constata-se que para aqueles com escolaridade entre o e 4 anos, todos os fatores variaram de forma a retirá-los do estoque total de desempregados, tanto no Ceará como na RMF. Movimento semelhante ocorreu para o grupo entre 5 e 8 anos de estudo, em que somente a variação do peso desse grupo na PIA total da região, nesse caso todo o estado, não corroborou para a retirada do estoque total de desempregados, diferentemente da RMF, no qual todos os fatores tiveram efeitos positivos para retirá-los do desemprego.

TABELA 6: EVOLUÇÃO DA PARTICIPAÇÃO NO DESEMPREGO SEGUNDO OS ANOS DE ESTUDO - CEARÁ E REGIÃO METROPOLITANA DE FORTALEZA $2001 \mathrm{E} 2008$

\begin{tabular}{c|c|c|c|c|c|c|c|c}
\hline \multirow{2}{*}{ Descrição } & \multicolumn{2}{|c|}{ De o a 4} & \multicolumn{2}{c|}{ De 5 a 8} & \multicolumn{2}{c|}{ De 9 a 11 } & \multicolumn{2}{c}{ Acima de 12 } \\
\cline { 2 - 8 } & Ceará & RMF & Ceará & RMF & Ceará & RMF & Ceará & RMF \\
\hline$\Delta \ln \left(\frac{U_{I}}{U}\right)$ & $-0,235$ & $-0,622$ & $-0,128$ & $-0,383$ & 0,211 & 0,446 & 0,261 & 0,658 \\
$\Delta \ln \left(\frac{U_{i}}{N_{i}}\right)-\Delta \ln \left(\frac{U}{N}\right)$ & $-0,090$ & $-0,244$ & $-0,126$ & $-0,189$ & $-0,028$ & 0,071 & 0,069 & 0,272 \\
$\Delta \ln \left(\frac{N_{i}}{P_{i}}\right)-\Delta \ln \left(\frac{N}{P}\right)$ & $-0,031$ & $-0,100$ & $-0,016$ & $-0,062$ & 0,008 & 0,024 & $-0,014$ & $-0,003$ \\
$\Delta \ln \left(\frac{P_{i}}{P}\right)$ & $-0,114$ & $-0,277$ & 0,014 & $-0,132$ & 0,231 & 0,350 & 0,205 & 0,388 \\
\hline
\end{tabular}

Fonte: PNAD, 2001 - 2008 / IBGE

Para a PEA desocupada com escolaridade entre 9 e 11 anos, observou-se sua elevação no estoque total de desempregados tanto no estado quanto na RMF, sendo mais acentuado para essa do que para aquele. Na RMF, todos os fatores contribuíram para elevar a participação desse grupo no estoque total de desempregados, diferentemente do resultado observado para o estado, onde o comportamento da taxa de desemprego do grupo em comparação ao da taxa de desemprego do estado variou de forma a retirá-los do desemprego, sem, contudo, obter sucesso final, haja vista que os demais fatores tiveram maior intensidade em elevá-los no desemprego total.

Para a PEA desempregada com escolaridade de 12 ou mais anos, somente o 
comportamento da taxa de participação da força de trabalho do grupo em comparação com o da taxa de participação da força de trabalho na região movimentou-se no sentido de reduzir a participação da PEA no estoque total de desempregados, o que não foi suficiente, dado que ocorreu elevação desse grupo no estoque total de desempregados no estado e na área metropolitana.

\section{Considerações Finais}

Este artigo teve como objetivo observar a dinâmica recente do mercado de trabalho no estado do Ceará e na Região Metropolitana de Fortaleza, com foco específico sobre o desemprego. Os principais achados nos faz debruçar sobre um mercado de trabalho com forte incidência de informalidade, sendo que, em 2008, 71,6\% no estado e 56,8\% na RMF representavam o contingente de trabalhadores sem proteção trabalhista. Mesmo com o elevado nível de informalidade, o desemprego aberto ainda atingiu 8,7\% na RMF e 6,2\% no estado, nesse mesmo ano.

Como “regra”, o desemprego não só o metropolitano, mas também o estadual atingiram, com maior intensidade, a força de trabalho feminina, durante todos os anos analisados. Além do mais, a PEA não branca apresentou elevadas taxas de desemprego aberto, mesmo em um cenário de redução ao longo dos anos observados.

Além disso, observou-se que o desemprego é bastante elevado para a força de trabalho jovem, com idade entre 15 e 24 anos, tanto no tecido metropolitano quanto em todo o estado, atingindo 13,7\% da PEA jovem em nível estadual e $19,8 \%$ na RMF. Houve ainda reduzidas taxas de desemprego para a PEA com idade acima de 60 anos em toda a série observada e em ambas as áreas analisadas. Adicionalmente, constataram-se reduzidas taxas de desemprego para a PEA com baixo nível de escolaridade e taxas mais elevadas para aqueles com mais anos de estudo em todo o Ceará, bem como na RMF. Esse resultado pode indicar que os postos de trabalho criados no Ceará, em sua maioria, exigem mão-de-obra pouco qualificada, dado o tipo de empreendimentos/ atividades que predominam na RMF e em todo o estado.

Utilizando-se o método de composição do desemprego para o Ceará e a RMF, os dados mostraram que, no estado, ocorreu redução da participação da força de trabalho masculina no desemprego total e, por outro lado, elevação da participação da força de trabalho feminina no desemprego. Já na RMF, a dinâmica foi contraria, elevando-se a participação da força de trabalho masculina no desemprego total em detrimento da redução da força de trabalho feminina.

Em relação à raça/cor, no estado e na $\mathrm{RMF}$, bem como, para a força de trabalho branca e não branca, registrou-se redução da participação deles no estoque de desempregados, sendo mais significativa a redução para os brancos em 
detrimento dos não brancos. A intensidade da redução foi mais acentuada no âmbito estadual em relação ao metropolitano.

No tocante ao estoque de desempregados segundo a idade da PEA, constatou-se dinâmica diferenciada entre as faixas etárias e entre os espaços observados. É fato, porém, que se elevou a participação da PEA desempregada com idade entre 15 e 24 anos tanto na RMF quanto em todo o Ceará. Contrariamente, reduziu-se a participação daqueles que tinham idade entre 25 e 39 e 40 e 59 anos, tanto em escala estadual quanto metropolitana.

No que diz respeito à composição do desemprego segundo os anos de estudo, os dados mostram que todos os fatores observados contribuíram para retirar a PEA desempregada, com escolaridade entre o e 4 anos de estudo, do estoque total de desempregados, em todo o estado e na RMF. Na faixa de 5 a 8 anos de estudo, o movimento foi semelhante, sendo que somente na variação do peso desse grupo na PIA total da RMF, enquanto em nível estadual, não foi observado tal efeito. Já nas faixas de 9 a 11 anos, bem como de 12 ou mais anos, elevou-se sua participação no estoque total de desempregados, em ambos os lócus geográficos estudados.

Com os resultados encontrados, pode-se afirmar que há, portanto, seletividade e, portanto, "vulnerabilidade" para determinados grupos sociais, no mercado de trabalho cearense e em sua RMF, isto porque o desemprego atinge em maior escala os grupos sociais compostos por mulheres, não brancos, jovens e, contrariamente ao esperado, a PEA com melhor nível de escolaridade.

\section{Referências Bibliográficas}

APARÍCIO, C. A. P.; QUEIROZ, S.N de. (2011). "Mercado de trabalho pós-crise: comparação entre as regiões metropolitanas de São Paulo e Fortaleza - 2009 e 2010." In: Anais do XII Encontro Nacional de Estudos do Trabalho. ABET, João Pessoa - PB.

BALTAR, P. E. de.A. (2003). "Estrutura econômica e emprego urbano na década de 1990.” In: PRONI, W. M.; HENRIQUE, W. Trabalho, mercado e sociedade. $O$ Brasil nos anos 90. São Paulo: editora UNESP, Campinas, São Paulo: Instituto de Economia da UNICAMP.

BARROS, R. P.; CAMARGO, J. M.; MENDONÇA, R. (1997). “A estrutura do desemprego no Brasil.” Texto para Discussão, n. 478, IPEA - RJ.

BASTOS, R. L. A. (2010). Desemprego Metropolitano no Brasil -1999-2007. Encontro Nacional da Associação Brasileira de Estudos Populacionais - ABEP. Anais... Caxambú - MG.

CANO, W. (1997). "Concentração e desconcentração econômica regional no Brasil: 1970/95.” Economia e Sociedade, Campinas, n.8, pp.101-141.

CORBACHO, A. (2000). "Labor markets in Central America: informal versus for- 
mal sectors”. Harvard Institute for International Development, development Discussion Papers, n. 747.

CORSEUIL, C. et al. (1997). "Determinantes da evolução da estrutura do desemprego no Brasil: 1986-1995.” Economia Aplicada. São Paulo: USP, v. (3), pp. 443-467.

CORSEUIL, C. et al. (1999). "Desemprego regional no Brasil: uma abordagem empírica.” Revista de Economia Aplicada, v. 3(3), pp. 407-435.

DEDECCA, C. S. (1998). "Reestruturação produtiva e tendenicas do emprego.” In: OLIVEIRA, A. M. (org). Economia e Trabalho, pp. 163-185, Campinas: IE/ Unicamp.

DINIZ, C. C. (1995). "A dinâmica regional recente da economia brasileira e suas perspectivas.” Texto para Discussão $n^{\circ} 375$. Brasília: IPEA.

FREIJE, S. (2001). "El empleo informal en America Latina y el Caribe: causas, consecuencias y recomendaciones de política”. Banco Interamericano de Desarrollo, Primer Seminario Tecnico de Consulta Regional sobre Temas Laborales.

FUNKHOUSER, E. (1996). "The urban informal-sector in Central America: Household survey evidence.” World Development, v. 24 (11), pp. 1.737-1.751.

HIRATA, H. (2009). “A precarização e a divisão internacional e sexual do trabalho.” Revista Sociologias. Porto Alegre, ano 11, $\mathrm{n}^{0}$ 21, pp. 24-41.

IPECE. (2011). Estimativa do PIB cearense em 2010 e seu desempenho setorial. URL [on-line]: < http://www.ipece.ce.gov.br/publicacoes/ipece-informe/IPECE_Informe_No2_PIB_2010.pdf >. Acesso em: 07 jul. de 2011.

JATOBÁ, J.; ANDRADE, E. G. L. de. (1993). "Desregulamentação do Mercado e das Relações de Trabalho no Brasil: potencial e limitações.” Texto para Discussão $n^{o} 312$, Brasília, IPEA.

MARCOULliER, D.; RUIZ de CASTILLA, V.; WOODRUFF, C. (1997). "Formal measures of the informal-sector wage gap in Mexico, El Salvador and Peru." Economic development and cultural change, v. 45(2), pp. 367-392.

MÁRQUEZ, G.; PAGÉS, C. (1998). "Ties that Bind: Employment Erotection and Labor Market Outcomes in Latin America.” Working Paper 373. Washington: Inter-American Development Bank.

MTE-DIESSE. (2007). Aspectos Conceituais da Vulnerabilidade Social. URL [on-line]: <http://www.mte.gov.br/observatorio/sumario_2009_TEXTOV1.pdf $>$. Acesso em 10 jul. de 2011.

MIRO, V. H.; SULIANO. D. C. (2010). Ceará: terra da luz para igualdade racial? VI Encontro de Economia do Ceará em Debate, Anais...Fortaleza, 20 p.

OZORIO de ALMEIDA, A. L.; ALVES, L. F.; GRAHAM, S. M. (1995). Poverty deregulation and employment in the informal sector of Mexico. World bank, education and social police department.

PACHECO, C. A. (1999). "Novos padrões de localização industrial? Tendências recentes dos indicadores da produção e do investimento industrial." Texto para Discussão $n^{\circ} 633$.

POCHMANN, M. (1998). Desemprego e politicas de emprego: tendências internacionais e o Brasil. In: DE OLIVEIRA, A. M. (org). Economia e Trabalho, pp. 219-233, Campinas, IE/Unicamp. 
POCHMANN, M. (1999). O trabalho sob fogo cruzado: exclusão, desemprego e precarização no final do século. São Paulo: contexto.

PONTES, P. A.; VIANNA, P. J. R.; HOLANDA, M. C. (2006). “A Política de Atração de Investimentos Industriais do Estado do Ceará: uma análise do período de 1995 a 2005." Texto para Discussão, Fortaleza, Dezembro.

RAMOS, L. (2002). “A evolução da informalidade no Brasil metropolitano: 19912001." Texto para Discussão $n^{\circ} 914$.

REMY, M. A. P. de.; QUEIROZ, S. N. de.; SILVA FILHO, L. A. da. (2010). "Evolução recente do emprego formal no Brasil: 2000-2008.” In: Anais do XVII Encontro Nacional de Estudos Populacionais. ABEP, Caxambu, MG.

SILVA FILHO, L. A.; QUEIROZ, S. N. de. (2009). “A Trajetória da Indústria e do Emprego Formal no Ceará 1996/2006.” In: Anais do XI Encontro Nacional de Estudos do Trabalho. ABET, Campinas - SP. . (2009). Política de concentração ou desconcentração industrial no Ceará?. Revista de Administração Pública e Gestão Social, v.1 (4), pp. 1-22, out./dez.

. (2011). "Recuperação econômica e emprego formal: avaliação empírica para o Nordeste brasileiro - 2000/2008." Revista Perspectiva Econômica, v. 7(1), pp. 42-54.

SILVA FILHO, L. A. (2011). "Mercado de Trabalho e Estrutura Sócio-ocupacional: estudo comparativo entre as regiões metropolitanas de Fortaleza, Recife e Salvador - 2001-2008." Dissertação de Mestrado em Economia. Universidade Federal do Rio Grande do Norte.

Recebido em: 28 de novembro de 2012 Aceito em: 5 de maio de 2014 
